\title{
Implementing clinical practice changes in critical care: lessons learned in a national collaborative of over 60 ICU teams
}

\author{
Ruth Kleinpell ${ }^{1-3}$, Jerry J. Zimmerman ${ }^{3,4}$ \\ ${ }^{1}$ Rush University Medical Center, Chicago Illinois, USA \\ ${ }^{2}$ Vanderbilt University Medical Center, Nashville Tennessee, USA \\ ${ }^{3}$ Society of Critical Care Medicine, Chicago Illinois, USA \\ ${ }^{4}$ Seattle Children's Hospital; Harborview Medical Center, University of Washington, \\ School of Medicine Seattle Washington, USA
}

\begin{abstract}
Improving care in the intensive care unit (ICU) is a global area of focus for clinicians worldwide. The complexity of the ICU environment, compounded by multiple patient comorbidities and high acuity levels, makes the ICU a unique and challenging area of clinical practice. The essence of quality improvement — designing and implementing strategies to enhance care - fit well with the ICU setting. However, implementing clinical change is challenging, regardless of the clinical setting. A national collaborative targeting ICU settings was initiated which provided a number of lessons learned regarding implementing quality of care initiatives in critical care. This article highlights quality improvement strategies for implementing clinical practice changes in the ICU to enhance care, based on reports from over 60 ICUs participating in the collaborative.
\end{abstract}

Anaesthesiology Intensive Therapy 2017, vol. 49, no 5, 437-440

Key words: critical care, quality, improvement; quality, improvement, collaborative

\section{OVERVIEW}

Over the recent years, all areas of healthcare have been charged to improve the quality and safety of healthcare. The emphasis in healthcare to decrease variations in care, promote best care practices including implementation of evidence based practice and adherence to clinical practice guidelines, and improve outcomes of care, have resulted in an increased focus on quality and performance improvement in critical care [1-3]. Improving clinical care practices requires focused efforts to plan, implement, and evaluate interventions aimed at promoting enhanced care in the intensive care unit (ICU). The literature on quality improvement highlights that the principles and practices of promoting changes in clinical care can be successfully achieved, yet challenges exist which require consideration. These include addressing strategies to change behavior, sustaining the focus on continuous improvement, and ensuring interdisciplinary leadership and collaboration [2].

\section{QUALITY IMPROVEMENT}

A number of quality improvement models and strategies exist that can be useful for clinical improvement initiatives. Among them, the Institute for Healthcare Improvement (IHI) offers a framework for instituting quality improvement initiatives that is easily adaptable to the ICU (Fig. 1) [4]. The IHI model for improvement is a strategy that many organizations have used to promote improvement strategies for clinical care. The continuous quality improvement (CQI) initiative uses a Plan-Do-Study-Act (PDSA) cycle (Fig. 1) to test a proposed change or CQI initiative. The cycle involves several key steps including 1) Forming a team to plan and implement the clinical practice change; 2) Setting the specific and measureable aims for what will be accomplished; 3) Establishing the outcome measures that will be evaluated if the aims are met; 4) Selecting the changes that need to be made to clinical practice routines; 5) Testing changes in clinical care to assess the impact on care; 6) After testing on a small scale, implementing changes in clinical 


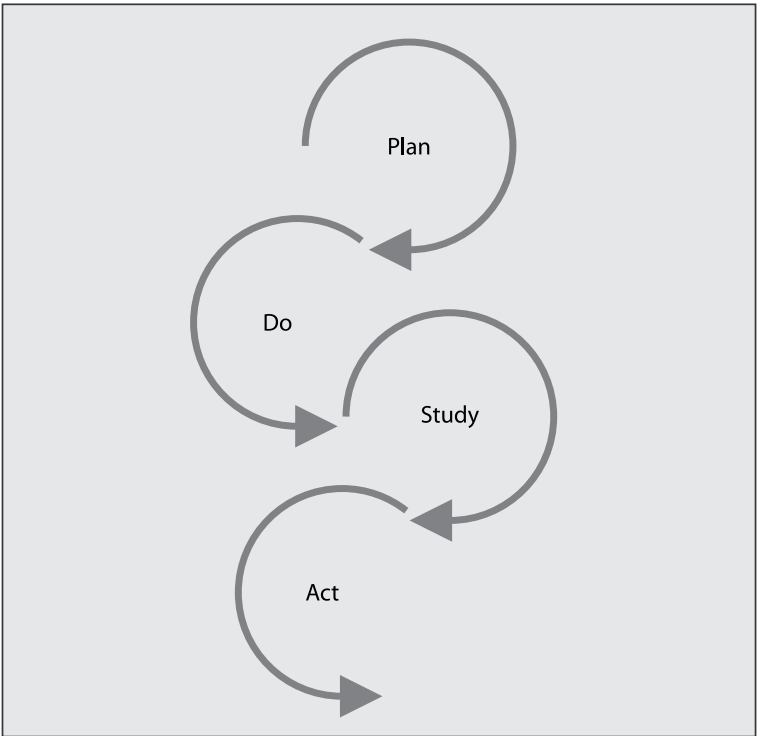

Figure 1. Institute for healthcare improvement (IHI) model for improvement

practice; 7) Spreading change after successful implementation of a clinical initiative for a pilot population or unit that can be disseminated to other parts of the organization [4]. Seminal guidelines for continuous quality improvement in the ICU highlight the importance of improving the level of performance of key processes in the ICU [5]. Among the essential components include establishing steps to improve performance of quality in the ICU, including structure (organizational aspects of the ICU); processes in the ICU, and specific outcomes such as length of stay, readmission rates, or patient satisfaction [5]. Quality improvement involves implementation of processes to monitor, assess, and improve an aspect of healthcare [6]. Hospitals in particular focus on quality improvement in order to improve clinical processes, enhance efficiency, and reduce costs of care [6, 7]. Additionally important components for a successful quality improvement initiative include motivation to change, teamwork, and leadership [2].

\section{QUALITY IMPROVEMENT IN CRITICAL CARE}

Initiating or improving quality improvement in critical care requires identification of opportunities for enhancement as well as building support [2]. Identifying potential barriers is an essential component in planning a quality improvement initiative. Often in clinical care, clinician resistance to change is a significant barrier to promoting practice changes. Acknowledging and addressing clinician resistance is an important consideration to instituting quality improvement in the ICU. Equally important are processes to support data collection, implementation, and evaluation [2]. A number of ICU quality indicators have been identified including unplanned readmissions, nosocomial infections,

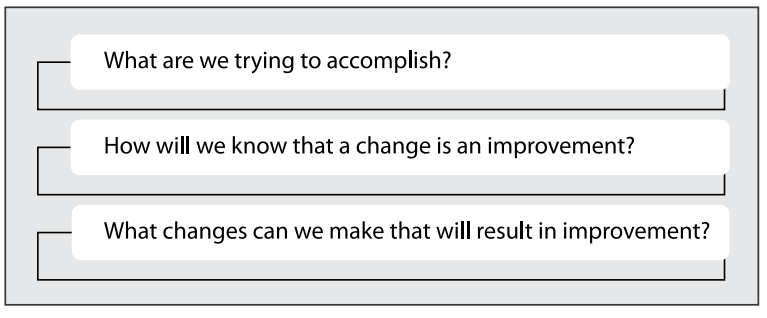

Figure 2. Intensive care unit improvement collaborative components

complication rates of diagnostic procedures, symptom management, and family satisfaction, among others $[2,3,5]$.

Targeting process improvement of clinical care in the ICU requires knowledge of relevant metrics, recommended best practices, and current clinical practice guidelines. As part of an improvement program targeting ICU care, a national collaborative was launched to assist ICU teams with implementing a patient centered care initiative. Spearheaded to address implementation of family centered care in the ICU [8], the collaborative was formed to create partnerships with other organizations promoting the same improvement work. Supported with national funding, the collaborative enabled 63 ICU teams to design, plan, and implement an improvement initiative (Fig. 2). The 63 teams represented both adult and pediatric ICUs in academic and community hospital settings, from 38 of the 50 United States. Over a 10 month timeline, the teams implemented a number of initiatives including open visitation, families on rounds, family information pamphlets, patient and family diaries, family care conferences, family advisory boards, and music therapy, among others (www.sccm.org/pcor-icu).

Several intentional components were integrated into the collaborative to provide ongoing support to the teams. An initial collaborative "kick-off" meeting which brought the teams together, helped to establish organizational commitment and outline key steps in implementation. Monthly coaching calls were held to assist the teams through the phases of project planning and implementation. In addition, quarterly webinars were held to highlight project implementation strategies and to address potential barriers to instituting clinical change. A number of "assignments" were used to help facilitate project work, including identifying strategies being used to address challenges to project implementation, sharing of how data was being collected to demonstrate impact of the initiative, and identifying short and long term project goals.

In addition, a monthly newsletter was provided to teams with collaborative related updates, reminders of upcoming calls and web conferences, and resources for project implementation and sustaining change. An electronic or "e" community listerve helped to promote team sharing as well as provide a mechanisms for dissemination of resources 
including articles of interest, sample tools and data collection forms, and relevant research studies.

\section{CHALLENGES TO IMPLEMENTING QUALITY IMPROVEMENT INITIATIVES IN CRITICAL CARE}

The 63 ICU teams participating in the national collaborative identified a number of barriers to project implementation including lack of buy-in from staff due to anticipated work/time required for the initiative, lack of staff awareness and interest, and issues with change readiness (Table 1). These reported barriers are not unique to critical care initiatives, and have been cited in the literature on quality improvement implementation [2,3]. Strategies that were identified by the teams to address barriers to promoting clinical change included forming a core project implementation team that met monthly to review the project status and plan next steps, enlisting the support of unit based champions, circulating email and other staff communications to provide project updates, and reinforcing the importance of the initiative by the ICU director and key unit leadership personnel.

\section{LESSONS LEARNED FROM A NATIONAL ICU COLLABORATIVE}

Several key lessons learned in implementing critical care quality initiatives were reported by the ICU teams including the usefulness of regular project team meetings that involve senior leaders to keep enthusiasm \& momentum, the benefit of ongoing communication to provide updates on the status of the project, clinician education, and reporting project successes, including patient and family feedback (Table 2). As highlighted in several "how-to" guides for quality improvement, enlisting stakeholder support, engaging unit based champions, highlighting benefits of the clinical practice change, positive messaging, and sharing progress metrics can help ICU teams to successful implement improvement initiatives (Fig. 3) [7, 8]. The collaborative format proved to be a beneficial way to assist a large number of ICU teams to simultaneously progress through the stages of implementing a quality improvement initiative. The collective sharing of experiences, including barriers faced and strategies used to address them, was instrumental in providing reassurance to the teams that progress can be made to make improvements in ICU care. A Tazmanian proverb highlights well the achievements that can be made through quality improvement initiatives - "little by little, a little becomes a lot" (http://www.inspirationalstories.com/proverbs/tanzanianlittle-by-little-a-little-becomes-a/)

\section{SUMMARY}

Quality improvement is an important component of improving care in the ICU. Identifying opportunities for initiating or improving an interdisciplinary quality improvement program is beneficial for promoting best outcomes for ICU patients and family members. Using a collaborative format to engage ICU teams to plan, implement, and evaluate a quality improvement initiative can be a useful way to assist ICU teams to enhance the quality of care in the ICU.

\section{ACKNOWLEDGEMENTS}

1. Source of funding: Patient-Centered Outcomes Research Institute (PCORI) Eugene Washington PCORI Engagement Award (6262-SCCM). Improving Care for Critically III Patients \& Families through Research Dissemination/ /Implementation.

2. Conflict of interest: none.
Table 1. Barriers to implementation of critical care clinical improvement initiatives

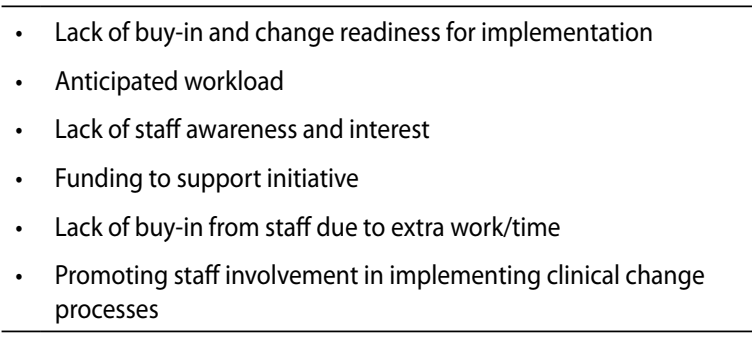

Table 2. Lessons learned in implementing critical care quality initiatives

- Ensure routine scheduled meetings that involve senior leaders to keep enthusiasm \& momentum

- Promote ongoing communication on project status

- Obtain buy-in from ICU providers to support clinical change

- Work to change culture within the unit

- Report on project implementation successes

- Share patient/family feedback to reinforce importance of initiative

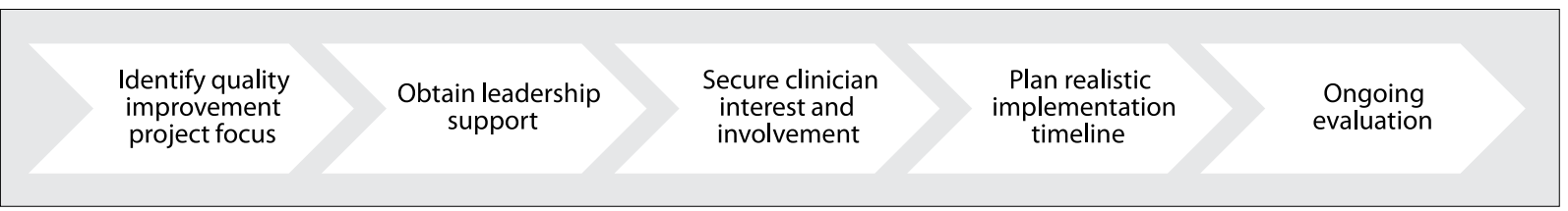

Figure 3. Strategies to engage ICU teams to implement improvement intiatives 


\section{References:}

1. Chelluri LP. Quality and performance improvement in critical care. Indian J Crit Care Med. 2008; 12(2): 67-76, doi: 10.4103/0972-5229.42560, indexed in Pubmed: 19742245.

2. Curtis JR, Cook DJ, Wall RJ, et al. Intensive care unit quality improvement: a "how-to" guide for the interdisciplinary team. Crit Care Med. 2006; 34(1): 211-218, indexed in Pubmed: 16374176.

3. Garland A. Improving the ICU: part 1. Chest. 2005; 127(6): 2151-2164, doi: 10.1378/chest.127.6.2151, indexed in Pubmed: 15947333.

4. Institute for Healthcare Improvement. Science of improvement: How to Improve 2011. http://www.ihi.org/knowledge/Pages/Howtolmprove/ ScienceoflmprovementHowtolmprove.aspx.

5. Thijs LG. Continuous quality improvement in the ICU: general guidelines. Intensive Care Medicine. 1997; 23(1): 125-127, doi: 10.1007/ s001340050304.

6. The National Learning Consortium. Continuous quality improvement strategies to optimize practice. 2013: 1-17.

7. Department of Health and Human Services Health Resources and Service Administration. Quality Improvement Guide. 2011.
8. HRSA Quality Improvement Toolkit. https://www.hrsa.gov/quality/ toolbox/methodology/qualityimprovement/index.html.

9. Davidson JE, Aslakson RA, Long AC, et al. Guidelines for family-centered care in the neonatal, pediatric, and adult ICU. Crit Care Med. 2017; 45(1): 103-128, doi: 10.1097/CCM.0000000000002169, indexed in Pubmed: 27984278.

\section{Corresponding author:}

Prof. Ruth Kleinpell

Rush University Medical Center

600 South Paulina Ave, Suite 1080

Chicago Illinois 60612, USA

e-mail:ruth_m_kleinpell@rush.edu

Received: 22.09.2017

Accepted: 4.11.2017 